\title{
The Utilization of Bovine Serum Albumin in the Preparation of Unicellular Organisms for Cytological Studies
}

\author{
Luis Oliveira', Hanh Huynh", Alan Burns ${ }^{2}$ and André Mackenzie ${ }^{2}$ \\ ${ }^{1}$ Department of Botany, The University of British Columbia, \\ Vancouver, B. C., V6T 2B1, Canada and 'Pulmonary Research Laboratory, \\ The University of British Columbia, Vancouver, B. C., Canada
}

Accepted June 6, 1988

The preparation of unicellular organisms for cytological research has always proved to be difficult because of the delicate nature of most cells. Of particular concern is the high percentage of broken cells and/or cellular distortions resulting from the need to concentrate the samples during preparation of the materials for cytological observation

Procedures aimed at solving or minimizing these problems can be subdivided into three major categories Filtration techniques (Bisalputra et al. 1973, Rittenburg et al. 1979, Palade et al. 1983), flat embedding of cells in an appropriate embedding vehicle (Kuhn 1981, Walker and Roberts 1982, Grenville 1983), and centrifugal concentration of biological materials (Dodge 1964, Hirsch and Fedorko 1968, Whitehouse et al. 1977, Outenreath and Davis 1985). Some of these techniques and their variations offer clear advantages for the handling of microorganisms in certain specific situations, while being unsuitable for more general purposes (e.g. Kuhn 1981, Walker and Roberts 1982, Asafo-Adjei et al. 1987).

In the case of multipurpose techniques some serious drawbacks exist. Filtration techniques produce compression and clumping of biological materials leading to cells touching one another over large segments of their surfaces (see Fig. 7 in Bisalputra et al. 1973). This makes the study of cell surface and associated phenomena (i.e. endocytosis and exocytosis) very difficult. Another major difficulty is the formation on filters of multiple layers of cells. These make cytochemical studies (i.e. localization of enzymatic activities) unreliable due to the heterogeneous exposure to the incubation media of cells occupying different layers. Centrifugation techniques are complicated by the loss of material experienced during the multiple concentration steps required for specimen preparation (Caceci and Herron 1985). The other major drawback is the care with which the sample must be kept warm during centrifugation to prevent premature solidification of the agar utilized in the embedment of cellular pellets (Hirsh and Fedorko 1968, Whitehouse et al. 1977).

In this work, we report on the use of bovine serum albumin (BSA) as a novel medium to prepare easy to handle pellets of unicellular organisms for cytological studies. This technique overcomes the problems mentioned above, while significantly reducing the time spent on the preparation of the materials. The procedure has the added advantage of allowing the same sample to be studied by different cytological methods. This makes data interpretation more reproducible and allows for an integrated approach to the study of microorganisms.

\section{Materials and methods}

\section{Organisms and culture conditions}

The microorganisms used in this study are summarized in Table 1. The media and the culture conditions used in the growth of marine organisms were similar to those described by Antia and Cheng (1970) and Oliveira and Antia (1984). Free swimming zoospores of Laminaria saccharina were obtained as described by Oliveira et al. (1980b). Growth and culture 
conditions for freshwater phytoplankters were reported by Vannini et al. (1978) for Euglena gracilis and by Daley and Brown (1973) for Synechococcus leopoliensis. Saccharomyces cerevisiae cells were grown aerobically at $28^{\circ} \mathrm{C}$ in liquid medium containing $1 \%$ yeast extract, $2 \%$ bacto-peptone and $2 \%$ pyruvate at $\mathrm{pH} 5.5$.

\section{Bovine serum albumin embedding}

Embedding and gelling of cellular materials in bovine serum albumin was accomplished by first concentrating by centrifugation $(3,000 \mathrm{~g}, 1 \mathrm{~min})$ a $10-\mathrm{ml}$ aliquot of material grown in liquid medium. The supernatant was discarded and $1 \mathrm{ml}$ of a $15-20 \%(\mathrm{w} / \mathrm{v})$ BSA solution in buffer or distilled water was added to the microorganism pellet. The cells were gently resuspended to mix them thoroughly with the BSA solution and recentrifuged. The pellet was

Table 1. Microorganisms used in the present study

\begin{tabular}{|c|c|}
\hline Species & Strain/clone \\
\hline \multicolumn{2}{|l|}{ MICROALGAE } \\
\hline \multicolumn{2}{|l|}{ Bacillariophyceae } \\
\hline Achnanthes brevipes (Agradh) Cleve & Watson No. 7 \\
\hline Skeletonema costatum (Greville) Cleve & Guillard "Skel" \\
\hline \multicolumn{2}{|l|}{ Bangiophyceae } \\
\hline Porphyridium cruentum Naegeli & Vischer No. 107 \\
\hline \multicolumn{2}{|l|}{ Chlorophyceae } \\
\hline Dunaliella tertiolecta Butcher & "Dun" \\
\hline Stichococcus bacillaris Naeg. & Local isolate \\
\hline Cryptophyceae & - \\
\hline Rhodomonas sp. & - \\
\hline \multicolumn{2}{|l|}{ Dinophyceae } \\
\hline Amphidinium carterae Hulburt & "Amphil" \\
\hline \multicolumn{2}{|l|}{ Euglenophyceae } \\
\hline Euglena gracilis Klebs & " $Z$ " \\
\hline \multicolumn{2}{|l|}{ Prymnesiophyceae } \\
\hline Hymenomonas elongata (Droop) Parke et Green & Millport No. 62 \\
\hline Pavlova lutheri (Droop) Green & Millport No. 60 \\
\hline Prymnesium parvum Carter & Millport No. 65 \\
\hline \multicolumn{2}{|l|}{ REPRODUCTIVE CELLS } \\
\hline \multicolumn{2}{|l|}{ Phaeophyceae } \\
\hline Laminaria saccharina (L.) Lamour & Zoospores \\
\hline \multicolumn{2}{|l|}{ CYANOBACTERIA } \\
\hline Synechococcus leopoliensis (Racib.) Komarek & UTEX No. 265 \\
\hline \multicolumn{2}{|l|}{ FUNGI } \\
\hline Saccharomyces cerevisiae Meyen ex Hansen (1883) & "DL1" \\
\hline
\end{tabular}

then gelled by cross-linking the BSA with $1 \%$ to $2 \%(\mathrm{v} / \mathrm{v})$ glutaraldehyde in buffer or aqueous solution (15 to $60 \mathrm{~min}$, room temperature or $4^{\circ} \mathrm{C}$ ).

The timing of the BSA embedding can be varied to meet the specific requirements of the study(ies) being conducted. The concentration of the glutaraldehyde and the period of crosslinking can be modified to control the consistency of the BSA gel. Gelled BSA blocks are washed in buffer, and the excess of BSA trimmed with a clean razor blade. The remaining gel containing the cellular material is then sliced into smaller portions that can be simultaneously subjected to diverse cytological treatments (Fig. 1).

\section{Histochemical studies}

Materials for histochemical studies were processed as previously described by Burns et al. (1982); that is, cells were fixed as for electron microscopy with omission of the post-fixation step. 


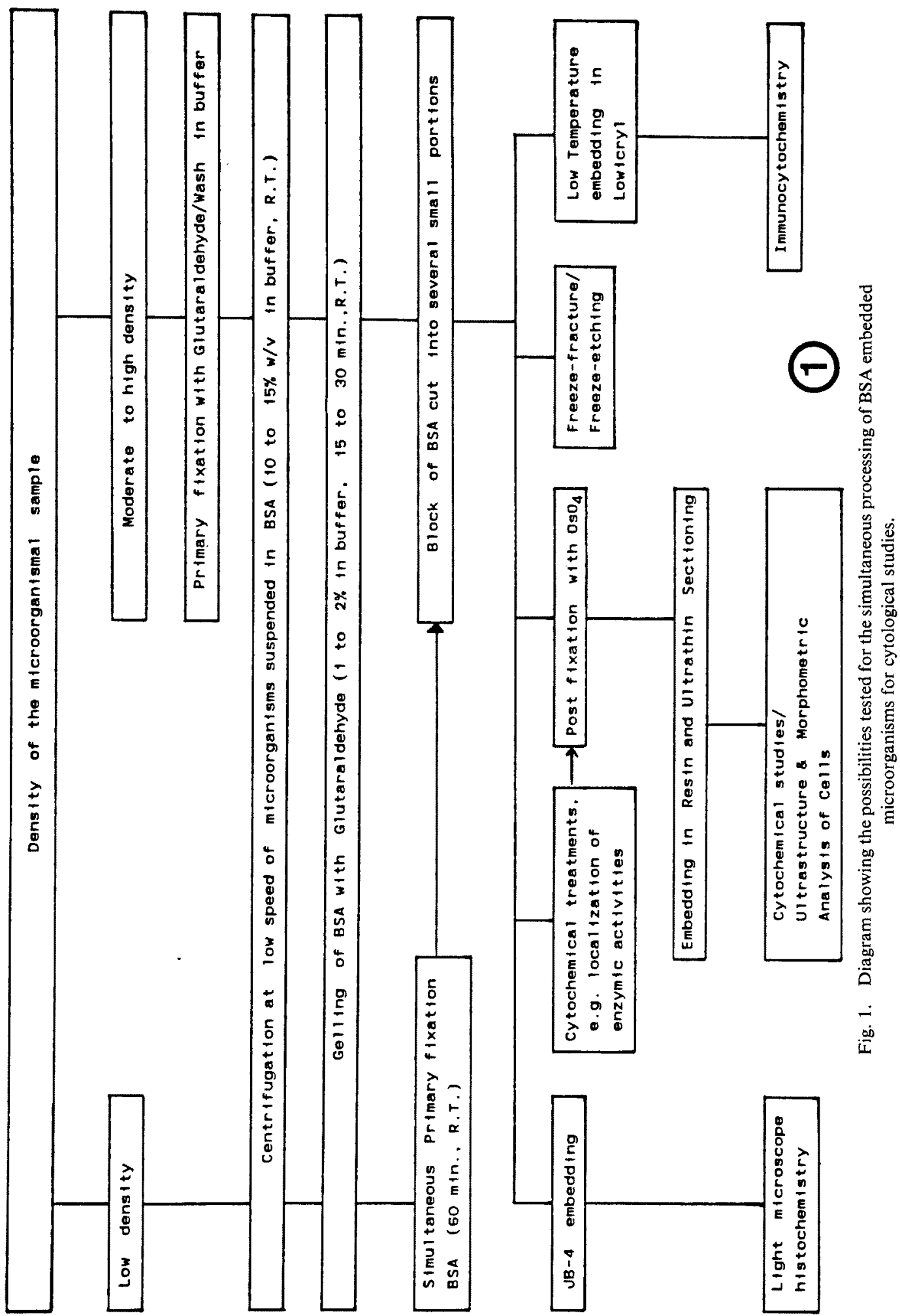




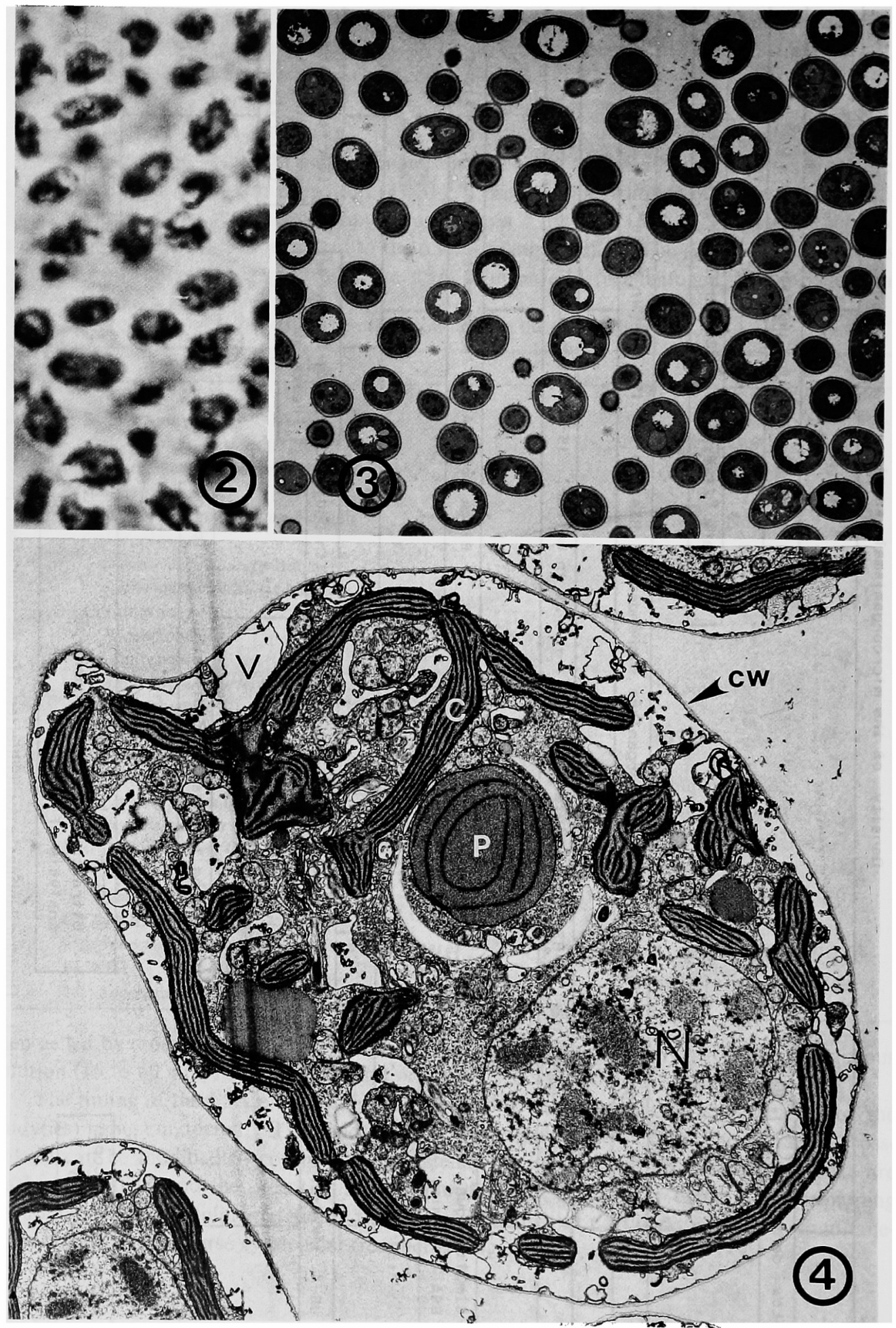


This was followed by the embedding of the material in JB-4 medium (Polyscience, Warrington, Pennsylvania). Thick sections (1 to $2 \mu \mathrm{m}$ ) were floated on to distilled water on clean microscope slides, air dried and stained. The staining procedures used included Toluidine Blue $O$ (TBO, pH 6.8) for carboxyl and sulphate groups (Evans and Holligan 1972), Aniline Blue Black for proteins (Fisher 1968), and Periodic Acid-Schiff's reaction for 1,2-glycol groups (Feder and O'Brien 1968).

\section{Transmission electron microscopy}

Primary fixations were carried out in 1 to $2.5 \%(\mathrm{v} / \mathrm{v})$ glutaraldehyde in $0.1-0.05 \mathrm{M}(\mathrm{w} / \mathrm{v})$ sodium cacodylate or saline phosphate buffers, $\mathrm{pH} 7.2-7.4$, for $90 \mathrm{~min}$., at room temperature or $4^{\circ} \mathrm{C}$. After repeated and thorough washing in buffer, post-fixation, when required. was conducted in $1 \%(\mathrm{v} / \mathrm{v})$ solution of osmium tetroxide in the same buffers for $60 \mathrm{~min}$ at room temperature or $4^{\circ} \mathrm{C}$.

After post-fixation, the material was rinsed in buffer and dehydrated through a series of aqueous ethanol or methanol solutions. These steps did not affect the BSA gelled blocks which were subsequently embedded in Spurr's (Spurr 1969) or PolyBed (Epon) 812 (Luft 1961) embedding resins.

\section{Cytochemical studies}

Cytochemical treatments were conducted both prior and after BSA embedding. No noticeable differences were observed between these two approaches. The cytochemical procedures included: Alcian Blue staining for the demonstration of the cell coat of the wall-less green flagellate Dunaliella tertiolecta (Oliveira et al. 1980a) and the localization of peroxidase activity (EC 1.11.1.7) in Stichococcus bacillaris (Oliveira and Bisalputra 1976).

\section{Freeze-etching studies}

Small blocks of glutaraldehyde fixed BSA-embedded material were washed in buffer and transferred to $25 \%$ glycerol in distilled water for an additional $2 \mathrm{hrs}$ period. The material was quenched in Freon 22 prior to freezing in liquid nitrogen. The frozen BSA-embedded material was then fractured, etched and coated in a Balzers BA $360 \mathrm{M}$ device according to the method described by Moor and Mühlethaler (1963).

\section{Results and discussion}

A technique to handle on a routine basis large numbers of samples and specimens of microorganisms for cytological observations must simultaneously satisfy several critical requirements, such as: (i) minimize compression and clumping of the cellular samples, (ii) minimize alterations in the preservation of cellular structure, (iii) speed up the processing of materials, (iv) prevent loss of cells during the preparative procedure, and (v) allow for the study of the same sample by different cytological methods.

Centrifugal "pelleting" of cells can lead to morphological distortion and even lysing of fragile cells due to clumping and compression of the biological materials (Bisalputra et al. 1973).

Fig. 2. Light microscope observation of sample of Amphidinium carterae stained with TBO. Observe the absence of signs of compression and/or clumping of cells.

Fig. 3. Low magnification electron micrograph of a sample of the yeast Saccharomyces cerevisiae. Observe the absence of signs of compression and/or clumping of cells.

Fig. 4. Electron micrograph of the marine dinoflagellate Amphidinium carterae. Observe the excellent preservation of cellular structure and absence of interference from the BSA background on the cell surface details. $\mathrm{C}=$ chloroplast, $\mathrm{CW}=$ cell wall, $\mathrm{N}=$ nucleus, $\mathrm{P}=$ pyrenoid, $\mathrm{V}=$ vacuole. 


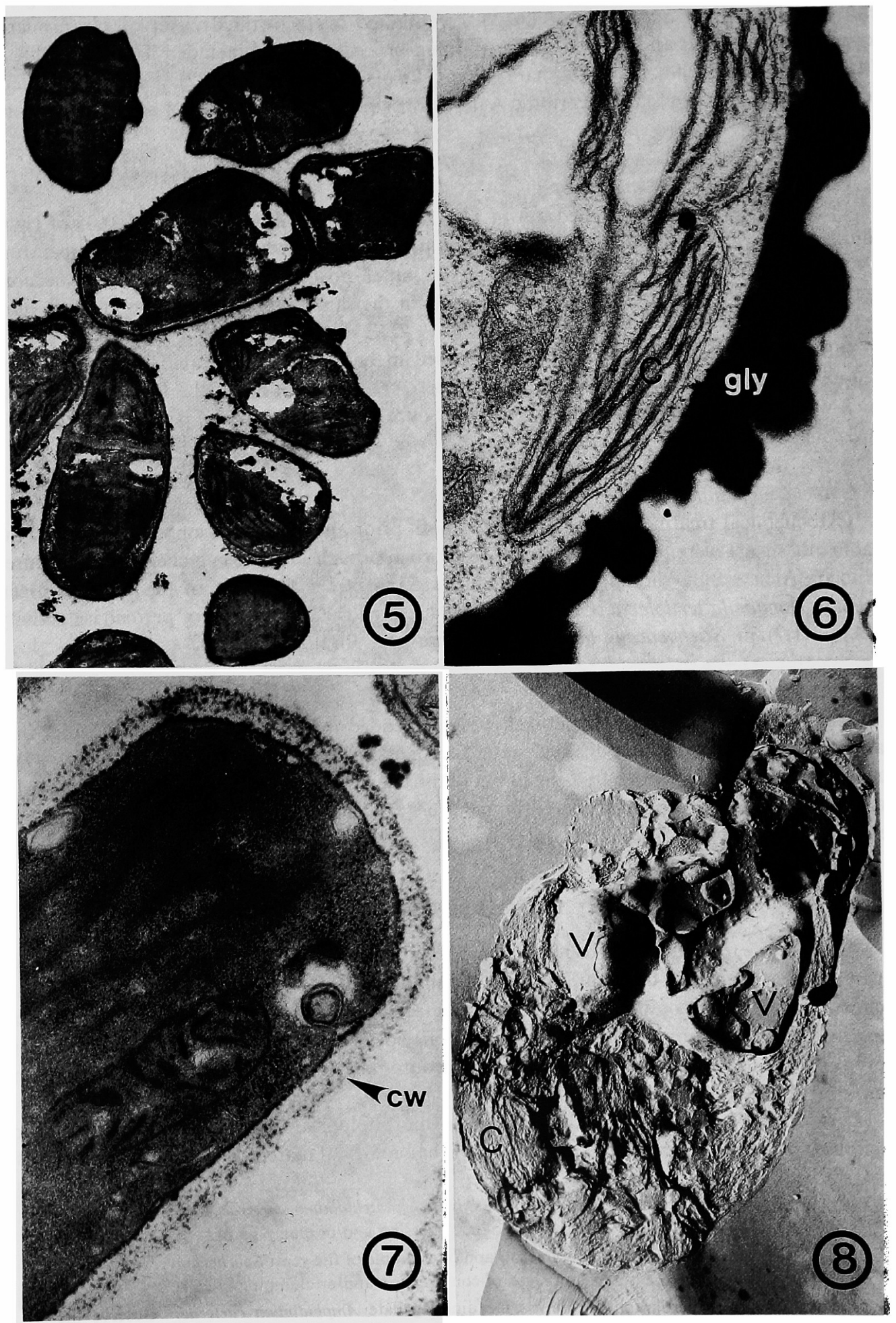


Clumping of biological materials has the added disadvantage of obscuring cell surface details. To minimize these problems, Palade et al. (1983) recently introduced the use of dextran as a nonosmophilic spacer to increase the distance between cells and prevent sample compression and clumping in materials concentrated by filtration. Figure 2 is a light micrograph of a sample of the marine phytoplankter Amphidinium carterae concentrated by centrifugation in BSA. The cells are well separated from one another revealing no signs of compression and/or clumping. This is confirmed by observation of sections of the yeast Saccharomyces cerevisiae with the electron microscope (Fig. 3). Important to notice is the fact that BSA embedding does not affect the sectioning and staining properties of the samples. It also produces a clear background that does not interfere with the preservation and observation of cytoplasmic structures (i.e. ribosomes, microtubules) and cellular organelles (i.e. mitochondria, chloroplasts) (Fig. 4). Another important advantage of this technique is that even fragile, highly vacuolated and thin walled cells, such as those of Amphidinium carterae, show no signs of disruption in preservation (Fig. 4). This indicates that BSA acts as a non-osmophilic spacer, as well as a shock absorber that minimizes the effects of the centrifugal forces; hence, the occurrence of distortions in cellular materials.

The processing of cells is further complicated by the loss of material experienced with repeated centrifugation during specimen preparation (Caceci and Herron 1985). This has restricted in the past the utilization of centrifugation to the use of samples of high cellular density (Glauert 1965) or to the introduction of special adaptations to handle samples with lower densities (Caceci and Herron 1985, Outenreath and Davis 1985, Hong and Barta 1986). Most frequently centrifugation of cellular suspensions is conducted in warm agar, obtaining on cooling a gelled pellet that can be subsequently handled as if it is a single block (Hayat 1972). However, the need to keep the samples warm to prevent premature gelling of the agar requires either properly fitted equipment (Whitehouse et al. 1977) or in its absence makes the whole procedure rather cumbersome. In contrast, the embedding of cells in BSA and subsequent gelling with glutaraldehyde is carried out at room temperature or even at lower temperatures if so required. This simplifies and speeds up the whole procedure by eliminating unnecessary preparative steps and removing temperature constraints. An added advantage is that gel consistency can be controlled by changing the concentration or the time of exposure to glutaraldehyde.

By adjusting the timing of BSA embedding, flexibility can be built into this technique to accommodate a variety of methodological requirements for cytological studies (Fig. 1). Samples of low cellular density can be handled without any difficulty by carrying out the pelleting stage prior to fixation. Under these circumstances glutaraldehyde acts simultaneously as a fixative for biological materials and as a cross-linking agent for BSA. This eliminates the need for special procedures and produces adequate concentrations for cytological observations (Fig. 5). Figure 6 shows the cytochemical staining of the wall-less microalga Dunaliella tertiolecta with Alcian Blue to visualize the glycocalyx-like cell coat characteristic of these organisms (Oliveira et al. 1980a). Localization of enzymic activities is also possible with this technique. Figure 7 shows the visualization of peroxidase activity in an unstained cell of Stichococcus bacillaris. Reaction product is found associated with the cell wall, plasma mem-

Fig. 5. Low magnification electron micrograph of a low density sample of Stichococcus bacillaris showing the adequate concentration of cells obtained with this technique.

Fig. 6. Cytochemical demonstration of the glycocalyx-like cell coat (gly) of the marine flagellate Dunaliella tertiolecta. $\mathrm{C}=$ chloroplast.

Fig. 7. Cytochemical demonstration of peroxidase activity in the chlorophycean alga Stichococcus bacillaris. $\mathrm{CW}=$ cell wall.

Fig. 8. Freeze-etching study of a sample of Amphidinium carterae. $\quad \mathrm{C}=$ chloroplast, $\mathrm{V}=$ vacuoles. 
brane and cristae of mitochondria. Incubation in the absence of hydrogen peroxide or treatment with potassium cyanide eliminates or strongly reduces the deposition of reaction product (not shown). Figure 8 represents a freeze-etching study of a cell of Amphidinium carterae. Important to notice is the ice-crystal free background produced by the BSA medium, a fundamental requisite in freeze-etching studies. The BSA procedure is also amenable to preparation of materials for light microscope histochemistry. Figure 2 shows cells of Amphidinium carterae stained with Toluidine Blue $O$ (Evans and Holligan 1972). Other histochemical stains, such as Aniline Blue Black (Fisher 1968) and Periodic acid-Schiff's reaction (Feder and O'Brien 1968), were also used successfully without any interference from the BSA background.

In conclusion it can be stated that BSA embedding provides a novel approach for the preparation of unicells for cytological observations and its suitability as a multipurpose technique was tested on a large number of different species of microorganisms (Table 1). Another important feature is that by subdividing the BSA-block into smaller segments, the same sample can be prepared for light microscopy (Fig. 2), transmission electron microscopy (Fig. 3), freeze-etching (Fig. 8) or any other technique (Fig. 1). This flexibility makes the correlation of data from different cytological observations more reproducible and less time consuming, since it avoids duplication of preparative steps.

\section{Summary}

Bovine serum albumin (BSA) is used as an embedding medium to produce pellets of unicellular organisms that can be handled as single blocks during preparation for cytological studies. The microorganisms are suspended in a solution of BSA, concentrated by gentle centrifugation and the BSA pellet gelled with glutaraldehyde. Gelling occurs at room temperature and its consistency can be controlled by changing the concentration or the time of exposure to glutaraldehyde. The part of the gelled block containing cellular material is then sliced into smaller portions that can be subjected to different cytological treatments. These make the correlation of data from different cytological observations more reproducible and less time consuming since it avoids duplication of preparative steps. BSA embedding also minimizes compression and clumping of cellular samples; hence, it minimizes artifacts in the preservation of cellular structure. The technique can be used to handle on a routine basis large number of specimens of microorganims for cytological observations.

Key words: BSA, cytology, embedding, microorganisms.

\section{Acknowledgement}

This work was supported by NSERC Grant A7844.

\section{References}

Antia, N. J. and Cheng, J. Y. 1970. The survival of axenic cultures of marine planktonic algae from prolonged exposure to darkness at $20^{\circ} \mathrm{C}$. Phycologia 9: 179-184.

Asafo-Adjei, E., Bernard, E. F. and Hase, T. 1987. Growing and embedding monolayer cells in situ in beem capsule caps for light and electron microscopy. J. Electron Microsc. Techn. 5: 367-372.

Bisalputra, T., Cheng, J. Y., Taylor, F. J. R. and Antia, N. J. 1973. Improved filtration techniques for the concentration and cytological preservation of microalgae for electron microscopy. Can. J. Bot. 51: 371377.

Burns, A. R., Oliveira, L. and Bisalputra, T. 1982. A histochemical study of bud initiation in the brown alga Sphacelaria furcigera. New Phytol. 92: 297-307.

Caceci, T. and Herron, M. A. 1985. Centrifuge adapters for processing small-volume, low concentration cell 
suspensions. J. Electron Microscopy Technique 2: 397-398.

Daley, R. J. and Brown R.S. 1973. Chlorophyll, nitrogen and photosynthetic patterns during growth and senescence of two blue-green algae. J. Phycol. 9: 395-401.

Dodge, J. D. 1964. Cytochemical staining of sections from plastic-embedded flagellates. Stain Technol. 39 : 381-386.

Evans, L. V. and Holligan, M. S. 1972. Correlated light and electron microscope studies on the brown algae I. Localization of alginic acid and sulphated polysaccharides in Dictyota. New Phytol. 71: 11611172.

Feder, N. and O'Brien, T. P. 1968. Plant microtechnique: some principles and new methods. Amer. J. Bot. 55: 123-142.

Fisher, D. B. 1968. Protein staining of ribboned epon sections for light microscopy. Histochemie 16: $92-96$.

Glauert, A. M. 1965. The fixation and embedding of biological specimens. In Techniques for Electron Microscopy. Edited by D. Kay and V. E. Cosslet. F. A. Davis Co., Philadelphia.

Grenville, D. 1983. A new method for flat embedding in glycol methacrylate. Stain Technol. 58: 57-58.

Hayat, M. A. 1972. Basic Electron Microscopy Techniques. Van Nostrand Reinhold Co., New York.

Hirsch, J. G. and Fedorko, M. E. 1968. Ultrastructure of human leukocytes after simultaneous flxation with glutaraldehyde and osmium tetroxide and "postfixation" in uranyl acetate. J. Cell Biol. 38: 615-627.

Hong, H. and Barta, J. R. 1986. Processing small biological specimens for transmission electron microscopy. J. Electron Microscopy Technique $3: 377-378$.

Kuhn, H. 1981. A simple method for the preparation of cell cultures for ultrastructural investigation. J. Histochem. Cytochem. 29: 84-86.

Luft, J. H. 1961. Improvements in epoxy resin embedding methods. J. Biophys. Biochem. Cytol. 9: 409412.

Moor, H. and Mühlethaler, K. 1963. Fine structure in frozen-etched yeast cells. J. Cell Biol. 17:609-628.

Oliveira, L. and Bisalputra, T. 1976. Studies in the brown alga Ectocarpus in culture: ultrastructural localization of enzymic activities. Can. J. Bot. 54: 913-922.

- and Antia, N. J. 1984. Evidence of nickel ion requirement for autotrophic growth of a marine diatom with urea serving as nitrogen source. Br. Phycol. J. 19: 125-134.

-, Bisalputra, T. and Antia, N. J. 1980a. Ultrastructural observation of the surface coat of Dunaliella tertiolecta from staining with cationic dyes and enzyme treatments. New Phytol. 85: 385-392.

-, Walker, D. C. and Bisalputra T. 1980b. Ultrastructural, cytochemical and enzymatic studies on the adhesive "plaques" of the brown algae Laminaria saccharina (L.) Lamour. and Nereocystis luetkeana (Nert.) Post. et Rupr. Protoplasma 104: 1-15.

Outenreath, R. L. and Davis, C. P. 1985. A simple microcentrifugation tube for electron microscopy. Stain Technol. 60: 56-58.

Palade, P., Saito, A., Mitchell, R. D. and Fleischer, S. 1983. Preparation of representative samples of subcellular fractions for electron microscopy by filtration with dextran. J. Histochem. Cytochem. 31: 971-974.

Rittenburg, J. H., Bayer, R. C., Gallagher, M. L. and Leavitt, D. F. 1979. A rapid technique for preparing microorganisms for transmission electron microscopy. Stain Technol. 54: 275-280.

Spurr, A. R. 1969. A low viscosity epoxy resin embedding medium for electron microscopy. J. Ultrastr. Res. 26: 31-39.

Vannini, G. L., Fasulo, M. P., Bruni, A. and Dall'Olio, G. 1978. Structural and developmental aspects during the greening process of Euglena gracilis treated with myomycin. Protoplasma 96: 335-349.

Walker, M. H. and Roberts, E. M. 1982. A thin film albumin method for encapsulating single cells for ultramicrotomy. Histochem. J. 14: 999-1001.

Whitehouse, R. L. S., Benichou, J. C. and Ryter, A. 1977. Procedure for the longitudinal orientation of rodshaped bacteria and the production of a high cell density of procaryotic and eucaryotic cells in thin sections for electron microscopy. Biologie Cellulaire 30: 155-157. 